\title{
THIN SILICON MEMS CONTACT-STRESS SENSOR
}

\author{
J. Kotovsky ${ }^{{ }^{*}}$, A.C. Tooker ${ }^{1}$, and D.A. Horsley ${ }^{2}$ \\ ${ }^{1}$ Lawrence Livermore National Laboratory, Livermore, California, USA \\ ${ }^{2}$ University of California, Davis, California, USA
}

\begin{abstract}
This thin, MEMS contact-stress (CS) sensor continuously and accurately measures time-varying, solid interface loads in embedded systems over tens of thousands of load cycles. Unlike all other interface load sensors, the CS sensor is extremely thin $(<150 \mu \mathrm{m})$, provides accurate, high-speed measurements, and exhibits good stability over time with no loss of calibration with load cycling. The silicon CS sensor, $5 \mathrm{~mm}^{2}$ and $65 \mu \mathrm{m}$ thick, has piezoresistive traces doped within a load-sensitive diaphragm. The novel package utilizes several layers of flexible polyimide to mechanically and electrically isolate the sensor from the environment, transmit normal applied loads to the diaphragm, and maintain uniform thickness. The CS sensors have a highly linear output in the load range tested $(0-2.4 \mathrm{MPa})$ with an average accuracy of $\pm 1.5 \%$.
\end{abstract}

\section{INTRODUCTION}

This work offers the first, thin, MEMS contact-stress (CS) sensor capable of accurate in situ measurements of time-varying, contact-stresses between two solid interfaces (e.g. in vivo cartilage contact-stress and body armor dynamic loading) across thousands of load cycles. This CS sensor measures loads only over its surface area, which is typically small compared to the contact area under study, unlike a force sensor in which the entire load is transmitted through the sensor. The CS sensor described here responds only to solid, normal loads, not to hydrostatic and/or shear loads.

Although similar in design to MEMS pressure sensors, the primary characteristic differentiating this CS sensor (other than the lack of a reference volume) is its thickness and package. CS sensors must be thin so that placement between two solid contacting surfaces will not induce changes in the load distribution to be measured. Attempts have been made to measure interface loads [1 -5], however, these sensors are typically incapable of extended measurements and are $>500 \mu \mathrm{m}$ thick. The CS sensor described here is extremely thin $(<150 \mu \mathrm{m}$ fully packaged $)$ so that it can be unobtrusively placed between contacting structures to provide accurate, long-term measurements.

All existing commercial technologies use polymer-based sensors. Although these commercially available sensors can be thin $(\sim 200 \mu \mathrm{m})$, since the sensing mechanism is the polymer material, the accuracy, speed, and longevity is limited by the timevarying, viscoelastic behavior of those materials. The CS sensor, in contrast, relies upon the phenomenal material properties of silicon, which shows no wear, is perfectly elastic, and has an exceptional gauge factor.

The two most commonly used commercial sensors for measuring interface loads are: the Fuji Prescale film (Fuji Photo Film Company, Tokyo, Japan) and the Tekscan FlexiForce sensor (Tekscan Corporation, Boston, MA). The Fuji Prescale film is comprised of dye-filled capsules that rupture when load is applied. The film produces a stain with an intensity proportional to the maximum applied load during the entire loading period. Analogous to photographic film, the material is only useful for a single exposure to load and is not relevant for embedded or dynamic applications.
The Tekscan FlexiForce sensor is comprised of two polyester sheets patterned with piezoresistive ink electrodes. Due to inelastic changes in the sensing ink, however, the Tekscan sensor is renowned for its loss of calibration over multiple load cycles, severely limiting its accuracy. This minimizes its utility for repeated, long-term load measurements.

The thin, MEMS CS sensor presented here provides a continuous, accurate contact-stress measurement. It is specifically designed to be extremely stable over the long-term, exhibiting no loss of calibration with load cycling. The CS sensor is built from elastic, well-characterized materials, providing accurate and highspeed $(50+\mathrm{kHz})$ measurements over a potential embedded lifetime of decades. This work explored sensor designs for an interface load range of $0-2.4 \mathrm{MPa}$; however, the CS sensor has a flexible design architecture to measure a wide variety of interface load ranges.

\section{CS SENSOR DESIGN AND FABRICATION Silicon MEMS CS Sensor Design}

This first-generation CS sensor is $2 \mathrm{~mm} \times 2.5 \mathrm{~mm}$ and is $65 \mu \mathrm{m}$ thick (Fig. 1). It is a MEMS-fabricated, silicon device with a load sensitive diaphragm. The sensor is similar in performance to established silicon pressure sensors; however, it is reliably produced down to a thickness of $50 \mu \mathrm{m}$.

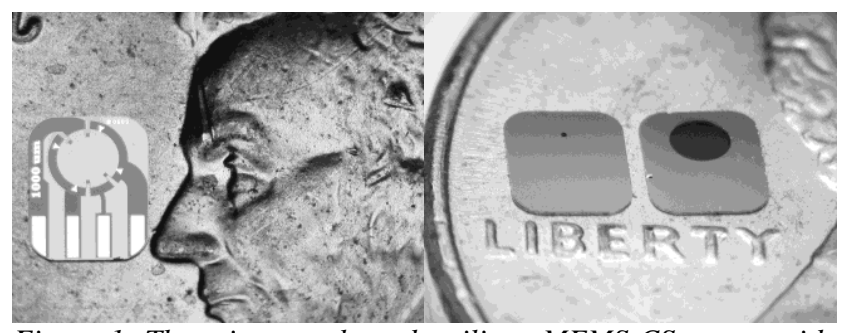

Figure 1: These images show the silicon MEMS CS sensor with a penny (for scale). The left image shows the front-side of a silicon MEMS CS sensor with a $500 \mu \mathrm{m}$ radius diaphragm. The right image shows the backside of two silicon MEMS CS sensors (50 $\mu \mathrm{m}$ and $500 \mu \mathrm{m}$ radius diaphragm, left and right, respectively) showing the etched diaphragms.

Four radially oriented piezoresistors are arranged in a full Wheatstone bridge at the edges of the diaphragm, where the tensile bending stresses are greatest, optimizing the signal-to-noise ratio (Fig. 2). Utilizing a full Wheatstone bridge, as well as the direction-dependent piezoresistive properties of silicon, the CS sensor is designed to be thermally compensating and selfbalancing, eliminating the need for a completion bridge. The piezoresistors lead to large metal-covered contact pads at the edge of the device for direct electrical connection to the package. The silicon CS sensors are designed to show no temperature or humidity dependence and to be drift-free with long-term use.

The load-sensitive, circular diaphragms are designed to be free of stress concentrations, making them mechanically strong and tolerant of $10 \mathrm{x}$ overloading. Diaphragm thicknesses of $0.5-25 \mu \mathrm{m}$ and radii of $50-500 \mu \mathrm{m}$ have been produced to accommodate load ranges for various applications. 


\section{Silicon MEMS CS Sensor Fabrication}

The silicon CS sensor is fabricated using a silicon-oninsulator (SOI) wafer with a device layer that defines the final diaphragm thickness. The SOI wafer enables reproducible control of the diaphragm thickness, regardless of the etch process or wafer thickness variation. Use of a thin buried-oxide layer minimizes the residual stresses on the finished diaphragms resulting in excellent sensor performance.

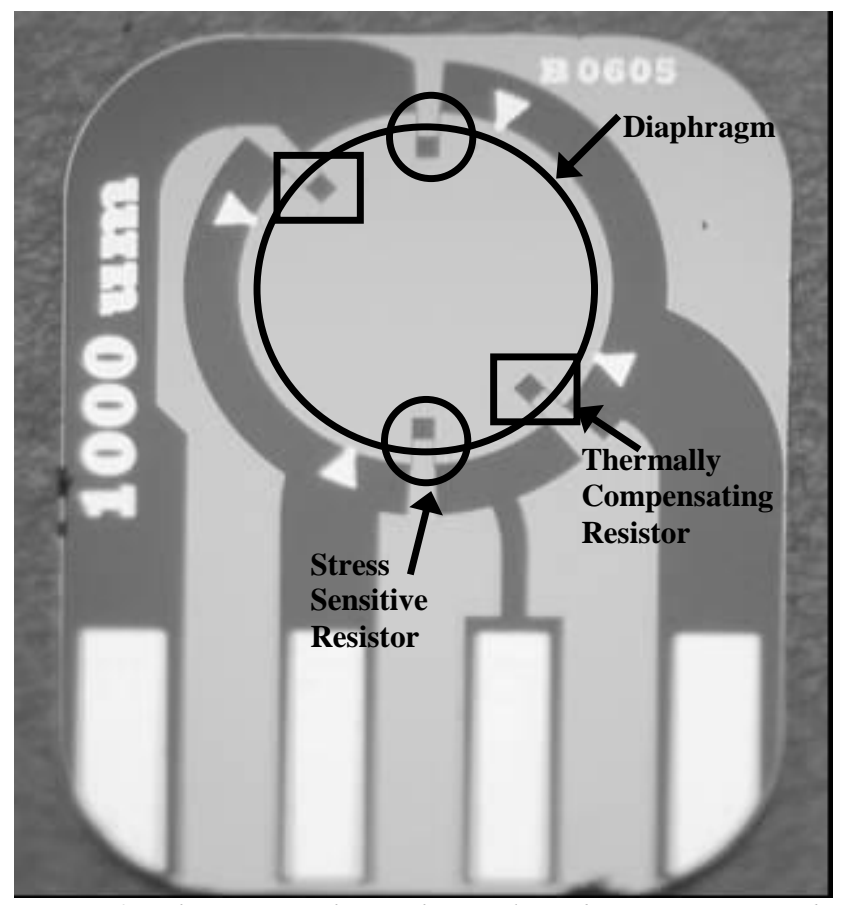

Figure 2: This image shows the implanted piezoresistors (dark gray) placement relative to the diaphragm location (large circle). The thermally compensating resistors (small boxes) and the stresssensitive resistors (small circles) are marked. At the bottom of the silicon sensor are four large, metal contact pads for connection to external electronics. This silicon CS sensor has a $500 \mu \mathrm{m}$ radius diaphragm. (The CS sensor has text "1000 um" denoting the diaphragm diameter and "B0605" denoting the sensor ID.)

An insulating layer is added to the device layer to electrically isolate the doped traces. Ion implantation into the device layer defines the piezoresistive Wheatstone bridge. The implant is annealed and windows are opened at the metal contact locations. Metal is patterned over the contact windows to form ohmic contacts with the implant.

The SOI wafer is then lapped to its final thickness of $65 \mu \mathrm{m}$ and mounted on a handle wafer for the final processing steps. The diaphragms are etched from the backside into the SOI wafer using a Deep Reactive Ion Etch (DRIE) process until the BOX layer is exposed, leaving only the device and insulating layers. The sensors are released after a second DRIE process etches through the entire wafer thickness to define the sensor outlines. Individual sensors are electrically tested to verify their piezoresistive response and then soldered to the package.

\section{CS Sensor Package}

In addition to the challenge of reliably producing a thin silicon MEMS sensor, the CS sensor demands a novel package utilizing several layers of flexible polyimide (DuPont, USA) to fully encapsulate the silicon sensor. The package provides a direct electrical connection between the silicon sensor and the external electronics while maintaining mechanical and electrical isolation from the environment (Figs. 3 and 4). The packaging is designed to maintain a uniform thickness $(<150 \mu \mathrm{m})$, including the region where the silicon sensor is mounted. The use of flexible, narrow polyimide strips ensures the packaged CS sensor can accommodate curved surfaces. The packaged CS sensor can withstand extreme loads without failure (no solder-joint failure) over tens of thousands of load cycles and survives repeated cycling between $-40{ }^{\circ} \mathrm{C}$ and $+70{ }^{\circ} \mathrm{C}$ while maintaining accuracy.

\begin{tabular}{|l|l|}
\hline Top Coverlay & $150 \mu \mathrm{m}$ \\
\hline Chip Shim & \\
\hline Bottom Coverlay & \\
\hline
\end{tabular}

Figure 3: A cross-section of the packaged CS sensor (not drawn to scale). The silicon CS sensor (in black) is soldered to the package and is completely encapsulated by the polyimide (Top and Bottom Coverlays and Chip Shim). The Bottom Coverlay has an electrodeposited copper layer with solder for making electrical connections to the sensor and the external electronics. The package maintains a uniform thickness of $<150 \mu \mathrm{m}$ and can be arbitrarily shaped to accommodate a variety of applications.
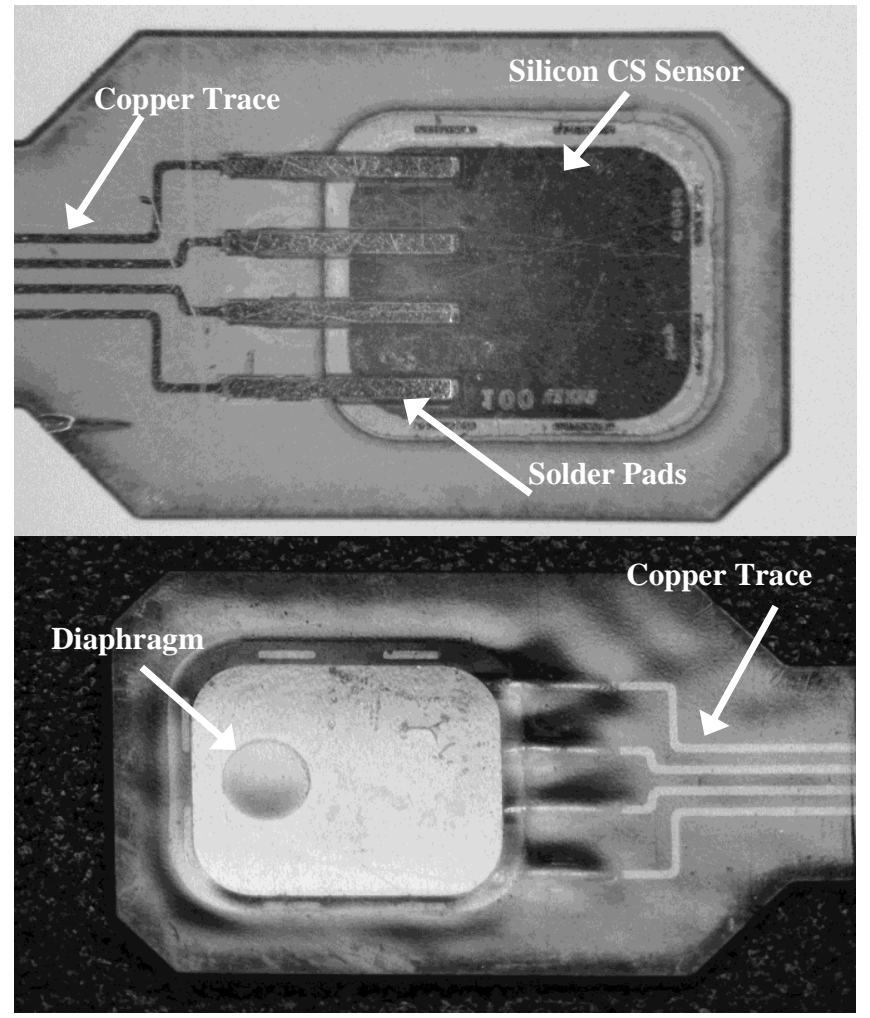

Figure 4: The top image shows the front-side of a packaged CS sensor. The silicon CS sensor is soldered to the package and the copper traces ensure electrical communication with the external electronics. The silicon CS sensor is completely encapsulated by the polyimide. The bottom image shows the back-side of a packaged CS sensor, including the etched diaphragm. 
The package is designed to transmit normal contact loads to the diaphragm, while isolating the silicon sensor from shear loads. In addition, the package is mechanically decoupled from the silicon sensor so that material property changes in the packaging materials do not adversely affect sensor performance. Finally, the package is patternable in arbitrary shapes for a wide array of applications.

\section{TESTING AND RESULTS}

\section{Testing Assembly}

Basic mechanical testing of the CS sensor is conducted using an Instron 5565 (Instron, Norwood, MA) fitted with 2 inch diameter flat, rigid plates. A compliant silicone rubber material is added between the two plates to mimic the contact environment (modulus and stiffness) of the sensor's application, although this layer is not required for sensor function. The Instron 5565 can be programmed to apply a variety of different load profiles. This particular testing assembly, including the load cell, limits the maximum load that can be applied to the CS sensor to approximately $2.4 \mathrm{MPa}$. Prior to testing, the CS sensors were conditioned by applying a minimum of 100 load cycles.

The standard input voltage to the Wheatstone bridge on the CS sensor is $12 \mathrm{~V} \mathrm{DC}$. Depending upon the application, though, any DC or AC voltage from $1-20 \mathrm{~V}$ can be used for the bridge input.

\section{Results}

Basic characterization data is presented here for a diaphragm thickness of $15 \mu \mathrm{m}$ and radius of $50 \mu \mathrm{m}$. Other diaphragm geometries were tested and exhibit similar results.

CS sensor drift was measured by applying and holding constant loads at $0 \mathrm{MPa}, 0.4 \mathrm{MPa}, 0.7 \mathrm{MPa}$, and $1.4 \mathrm{MPa}$. Drift is a measure of change of the output over time. With these constant loads, the maximum drift after 2 hours is $< \pm 0.7 \%$ of full-scale output (FSO). For no load $(0 \mathrm{MPa})$, the maximum drift after 18 hours is $< \pm 0.8 \%$ of FSO.

Figure 5 shows the CS sensor output for 5 load cycles (2429 data points) over a load range of $0.04-2.41 \mathrm{MPa}$. The typical CS sensor has an excellent linear calibration curve, shown in Fig. 6, with a coefficient of determination $\left(\mathrm{R}^{2}\right)>0.99$. (The coefficient of determination is calculated from the ratio of the regression sum of squares to the residual sum of squares in the least squares fit calculation.) Calibration curves with $\mathrm{R}^{2}>0.99$ can be found for the diaphragm geometries tested, however, for larger diaphragms the curves are typically non-linear. The linearity of the smaller-diaphragm CS sensors is advantageous for the simplicity in both use and calibration.

The calibrated sensor is then subjected to known load cycles and the measured response is compared to the applied load (Fig. 6). This second load cycling test, consisting of 15 load cycles (7297 data points) over a load range of $0.04-2.41 \mathrm{MPa}$, determines the accuracy of the CS sensor after the calibration curve is established.

The absolute error is the maximum difference between the measured and predicted pressure; this represents the maximum measurement uncertainty. For this typical CS sensor, the absolute error is measured to be $0.17 \mathrm{MPa}$. The absolute accuracy of the device can then be described as the maximum absolute error divided by the full-scale range of the device. Thus, the absolute accuracy is $\pm 7.0 \%$ of FSO.

This absolute accuracy of $\pm 7.0 \%$ of FSO is based on the absolute error and, therefore, represents the greatest uncertainty of the CS sensor measurement. The CS sensor error can also be expressed as the average error; this provides a typical measure of the CS sensor performance, although it hides the extremes of the uncertainty. The average error is the difference between the average measured and predicted pressure. The average accuracy of the device can then be described as the average error divided by the FSO of the device. The average error is $0.04 \mathrm{MPa}$, corresponding to an average accuracy of $\pm 1.5 \%$ of FSO.

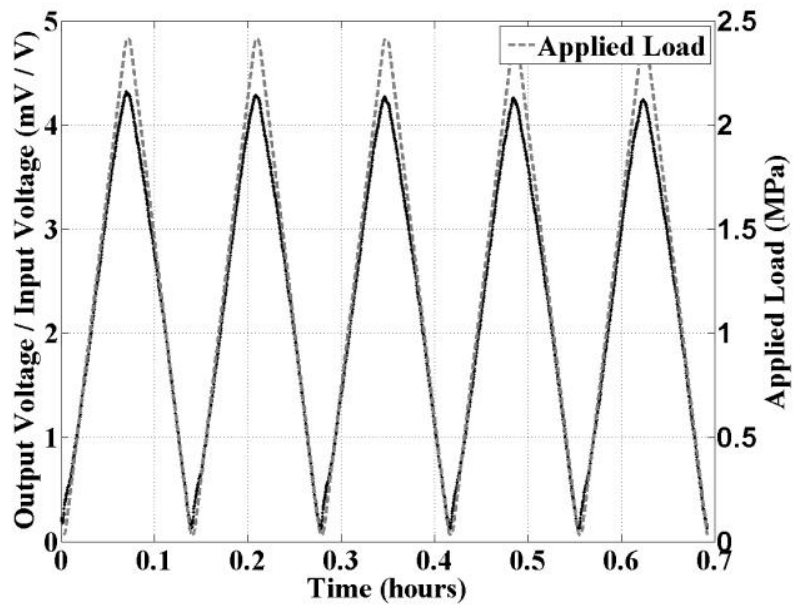

Figure 5: Graph of the CS sensor output (solid black line) for 5 load cycles (dashed gray line) over a load range of $0.04-2.41 \mathrm{MPa}$. The data shown is for a diaphragm thickness of $15 \mu \mathrm{m}$ and a radius of $50 \mu \mathrm{m}$.

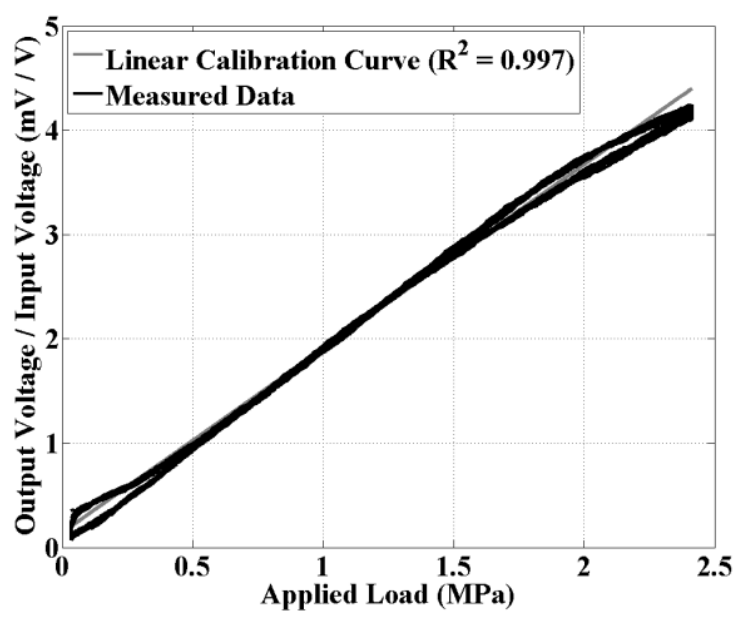

Figure 6: Graph of the linear calibration curve (thin gray line) for the 5 load cycles shown in Fig. 5. The measured data (thick black line) from a second load cycling test (15 load cycles) is also shown for comparison with the established calibration data. The average accuracy for this data, compared with the calibration data, is $\pm 1.5 \%$ of FSO, demonstrating the excellent performance of the CS sensor. The data shown is for a diaphragm thickness of $15 \mu \mathrm{m}$ and a radius of $50 \mu \mathrm{m}$; other diaphragm geometries exhibit similar performance.

The precision of the devices is calculated by looking at the standard deviation of the error. The standard deviation of the errors is $1.3 \%$, indicating the devices show excellent precision.

Hysteresis of the CS sensor is assessed by repeated loading and unloading of the sensor. The hysteresis is the largest difference in CS sensor output for increasing and decreasing loads 
(for the same applied load). As shown in Fig. 6, the CS sensors do exhibit small hysteresis, especially for smaller loads. For the typical CS sensor shown in Figs. 5 and 6, the hysteresis is $\pm 6.5 \%$ of FSO. The primary source of the hysteresis is in the testing assembly, as discussed below.

\section{DISCUSSION}

The CS sensors presented here have good accuracy and precision in the load range tested $(0.04-2.41 \mathrm{MPa})$. The load range tested was limited by the mechanical testing apparatus, not the CS sensors, themselves. Preliminary high-load tests, up to $4 \mathrm{MPa}$, and low-load tests, from $6.9 \mathrm{kPa}$, have been conducted and the CS sensors also demonstrate excellent performance for alternate load ranges.

\section{Calibration, Hysteresis, and the Testing Assembly}

Due to limitations of the testing system, the accuracy and hysteresis of the sensor are overstated. The mechanical testing assembly, unfortunately, contributes to the error and hysteresis calculations. The calibration of the CS sensors is affected by placement of the sensor in the testing assembly. During testing, it must be assumed that a uniform load distribution exists within the testing assembly. It is known, however, that variations in the load distribution exist. The compliant layer used in these experiments has a Poisson ratio of nearly 0.5 and is incompressible. As a result, the material shears and tends to extrude out of the testing platform. These effects limit the assumption of a uniform load distribution. Testing is performed at the center of the testing assembly to help minimize these effects. To control against this effect, a custom, fluid-diaphragm loading system is being created to produce a welldefined normal load to the device.

The compliant layer in the mechanical testing assembly is a viscoelastic material and its ability to mechanically "restore" itself on unloading is limited. As the load is increased and decreased, the viscoelastic behavior of the compliant layer affects the pressure distribution within the testing assembly, resulting in the observed hysteresis. Preliminary tests have shown that replacement of this compliant layer with a thinner or more elastic material minimizes this effect, thereby minimizing the hysteresis. In addition, maintaining a higher load during testing (i.e. testing from $0.40-2.41 \mathrm{MPa}$ instead of $0.04-2.41 \mathrm{MPa}$ ) further minimizes the hysteresis. Based on known material properties of the sensor and the testing assembly, as well as measurements that have shown no hysteresis, it is believed the hysteresis of the sensor itself is overstated.

\section{Bias}

The CS sensors have a full Wheatstone bridge. Ideally, the diaphragm would be in a zero stress-state with no applied load. With no stress, the four resistors comprising the bridge would be perfectly matched and, thus, the CS sensor output would be zero. Residual stresses in the diaphragm, however, produce a non-zero bridge output, even in a no-load state. This combined with minor variations in the resistors generated during fabrication can generate a non-zero output with no applied load (i.e. a DC bias). This DC bias has been subtracted from the CS sensor outputs shown in Figs. 5 and 6.

The DC bias values are typically between $5 \mathrm{mV} / \mathrm{V}$ and $30 \mathrm{mV} / \mathrm{V}$ and do not show a correlation to diaphragm geometry. Indeed, two CS sensors with the same diaphragm geometry often exhibit different DC bias values. This effect may be explained by stress variations across a wafer due to varied insulation film thicknesses. Although this DC bias does not affect the CS sensor performance or sensitivity, it necessitates individual calibration of the CS sensors. Further refinements in the film deposition and resistor design should minimize the film stress and resistor variations.

\section{SUMMARY AND FUTURE WORK}

The CS sensor presented here is a silicon-based MEMS sensor with piezoresistive traces strategically doped within a loadsensitive diaphragm. It is designed to repeatedly measure timevarying loads, with a high long-term accuracy. The silicon MEMS CS sensor can be reliably produced with thicknesses down to $50 \mu \mathrm{m}$ and the packaged CS sensor is $<150 \mu \mathrm{m}$ thick, ensuring the sensor minimally perturbs the interface loads of the system under study. The load sensitive diaphragms have been produced with radii between $50 \mu \mathrm{m}$ and $500 \mu \mathrm{m}$ and thicknesses between $0.5 \mu \mathrm{m}$ and $25 \mu \mathrm{m}$ to accommodate a variety of load ranges. The smaller diaphragm CS sensors have a highly linear response in the load range tested $(0-2.4 \mathrm{MPa})$. The sensors have an absolute accuracy between $\pm 7 \%$ of FSO, with an average accuracy between $\pm 1.5 \%$ of FSO.

The primary cause of the error and hysteresis for these CS sensors is in the mechanical testing assembly. A custom fluiddriven pressure testing assembly is being built to minimize the shearing effects, non-uniform loading, and hysteresis of the system described here. This will also enable testing of the next generation sensors at higher and dynamic loads, as well as varied temperatures. Currently, smaller, next-generation sensors, for both flat and curved environments, are in testing and very large (1000-element), flexible, stretchable stress-sensing arrays of similar design have been prototyped.

\section{ACKNOWLEDGEMENTS}

This work performed under the auspices of the U.S. Department of Energy by Lawrence Livermore National Laboratory under Contract DE-AC52-07NA27344. The technology is protected by Patent \#2060107752 and patents pending and is available for commercial license. LLNL Release Number: LLNL-PROC-427227.

\section{REFERENCES}

[1] M.L. Harrison, P. Morberg, W.J.M. Bruce, and W.R. Walsh, "An improved method for measuring tibiofemoral contact areas in total knew arthroplasty: a comparison of K-scan sensor and Fuji film", Journal of Biomechanics, 32, 951 (1999).

[2] M. Ferguson-Pell, S. Hagisawa, and D. Bain, "Evaluation of a sensor for low interface pressure applications", Medical Engineering and Physics, 22, 657 (2000).

[3] M. Manouel, H.S. Pearlman, A. Belakhlef, and T.D. Brown, "A miniature piezoelectric polymer transducer for in vitro measurement of the dynamic contact stress distribution", Journal of Biomechanics, 25 (6), 627 (1992).

[4] P.H. Chappell and J.A. Elliot, "Contact force sensor for artificial hands with a digital interface for a controller", Measurement Science Technology, 14, 1275 (2003).

[5] T.N. Tun, T.S. Lok, T.C. Jui, R. Akkipeddi, and M. Rahman, "Contact pressure measurement using silicon-based $\mathrm{Al}_{\mathrm{x}} \mathrm{Ga}_{1}$. ${ }_{x}$ As semiconductor pressure sensors", Sensors and Actuators A, 118, 190 (2005).

\section{CONTACT}

*J. Kotovsky, tel: +1-925-424-2398; kotovsky1@1lnl.gov 